\title{
Characteristics of Clinical Symptoms, Cerebral Images and Stroke Etiology in Vertebro-Basilar Artery Fenestration-Related Infarction
}

\author{
Nobukazu Miyamoto*® , Yuji Ueno®, Kenichiro Hira, Chikage Kijima, Sho Nakajima, \\ Kazuo Yamashiro and Nobutaka Hattori (D)
}

Department of Neurology, Juntendo University School of Medicine, 3-1-3 Hongo, Bunkyo-ku, Tokyo 113-0033, Japan; yuji-u@juntendo.ac.jp (Y.U.); ra-hi-.com@live.jp (K.H.); c-kijima@juntendo.ac.jp (C.K.); synakaji@juntendo.ac.jp (S.N.); kazuo-y@juntendo.ac.jp (K.Y.); nhattori@juntendo.ac.jp (N.H.)

* Correspondence: nobu-m@juntendo.ac.jp; Tel.: +81-3-3813-3111; Fax: +81-3-5800-0547

Received: 7 April 2020; Accepted: 19 April 2020; Published: 20 April 2020

\begin{abstract}
Cerebral artery fenestration is a rare variant of the vascular architecture, but its existence is well documented. The common site of fenestration is the vertebra-basilar artery and it may be found incidentally with subarachnoid hemorrhage. However, fenestration-related cerebral infarction is rare. We analyzed the clinical characteristics, stroke etiology, and image findings of fenestration-related cerebral infarction of the vertebrobasilar artery. We reviewed our hospital records and previously published reports to find cases of fenestration-related cerebral infarction. We excluded those with unknown clinical features or radiological findings. We retrieved 4 cases of fenestration-related infarction from our hospital, in which vascular change, headache, vertigo/dizziness, and dissection in stroke etiology were detected. In eight previously reported cases of fenestration-related infarction, similar vascular changes were noted, but they were mainly diagnosed as embolic stroke of undetermined source. However, based on the criteria for dissection in this study, dissection as the stroke etiology was suspected in the previously reported cases. Many hypotheses have been proposed for the development of dissection, thrombus, and aneurysms in fenestration. Although an embryological and morphological study is needed, clinicians must consider basilar artery fenestration-related infarction as a differential diagnosis and intensive non-invasive image study is recommended.
\end{abstract}

Keywords: fenestration; cerebral infarction; dissection; Magnetic resonance imaging (MRI); basi-parallel anatomic scanning magnetic resonance imaging (BPAS); embolic stroke of undetermined source (ESUS)

\section{Introduction}

Artery fenestration begins with a common origin and splits into two distinct endothelium-lined channels, which then rejoin distally. Cerebral artery fenestration is a rare but well-known vascular variation [1]. The fenestration size/length varies from $1 \mathrm{~mm}$ to several centimeters [2]. Fenestration in the cerebrovascular system was first detected in the vertebral artery during autopsy in 1866, and many cases have been reported since [1]. In post-mortem studies, basilar artery fenestration is present in $5.26 \%$ [3].

Conventional non-invasive imaging techniques, such as computed tomography angiography (CTA) and magnetic resonance angiography (MRA), are widely used for assessing the vascular structure, including fenestration, to provide information about adjacent structures and location, as well as the arterial lumen morphology. CTA image quality has significantly advanced, become more accurate and sensitive, and can incisively delineate the existence of cerebral vascular fenestration (11-12.9\%) [1,4]. In addition, CTA can accurately provide valuable information regarding the shape, 
size, and hemodynamic flow characteristics of cerebral arterial bifurcation, which change during aneurysm development. The first report of an aneurysm site in basilar artery fenestration by CTA was in 2003 [5]. Recently, using a large 5657 CTA series or 2280 MRA series, the shape, frequency, relationship with aneurysms, and anomalous origin of the anterior inferior cerebellar artery (AICA) in basilar artery fenestration were assessed [6,7]. More recently, a new three-dimensional (3D) black blood technique, called volumetric isotropic turbo spin-echo acquisition (VISTA) sequences, was developed, having the advantages of high multiple planar reconstruction and isotropic resolution, which helps to assess the wall nature of tangled intracranial arteries more accurately [8]. Using these methods, the plaques of basilar artery fenestration were demonstrated to exist mostly in the bifurcation and proximal segments [9].

In vertebrobasilar junction aneurysms, the frequency of basilar artery fenestration was reported to be 35-70\% [10,11]. Basilar artery fenestration-associated aneurysms are more frequent in the elderly [12]. Most reports have suggested that this vascular variation has no clinical symptoms in healthy people other than subarachnoid hemorrhage (SAH) [12]. In most patients with basilar artery fenestration, acute SAH is the initial presentation of an aneurysm at the vertebrobasilar junction [10]. There were only two cases in which fenestration was the only malformation that coexisted with SAH $[6,13]$. Thus, it is unclear whether fenestration itself causes the formation of intracranial aneurysms [14].

There are few case reports of fenestration-related cerebral infarction in the posterior circulation [15-17]. Therefore, the aim of this case series study was to clarify the stroke etiology, clinical characteristics, and image findings of fenestration-related cerebral infarction of the vertebrobasilar artery.

\section{Materials and Methods}

This study was conducted in accordance with the Declaration of Helsinki. The independent ethics committee of Juntendo University Hospital approved this study. Between January 2016 and December 2019, 813 patients with cerebrovascular diseases, including ischemic and hemorrhagic stroke, were admitted to the Department of Neurology, Juntendo University Hospital. We retrospectively investigated stroke patients who had been identified as having vertebra/basilar artery fenestration by Magnetic resonance imaging (MRI)/A. We also added basi-parallel anatomic scanning magnetic resonance imaging (BPAS) to the MRI protocol. BPAS can visualize the outer contours of the vertebrobasilar artery, even in the presence of occlusion. BPAS-MRI was performed on a 3.0-T scanner in 20-mm thick coronal sections parallel to the clivus using the fast spin-echo sequence.

For all cases from our hospital records, we recorded the patient's sex, age, National Institutes of Health Stroke Scale (NIHSS), vascular risk factors, such as hypertension (HT; systolic blood pressure (BP) of $>140 \mathrm{mmHg}$ or diastolic BP of $>90 \mathrm{mmHg}$, or drug treatment for HT), diabetes mellitus (DM; a glycated hemoglobin ( $\mathrm{HbA1}$ ) level of $>6.5 \%$ by the National Glycohemoglobin Standardization Program (NGSP) definition or drug treatment for DM), dyslipidemia (DL; a low-density lipoprotein (LDL) cholesterol level of $>140 \mathrm{mg} / \mathrm{dL}$, a high-density lipoprotein (HDL)-cholesterol level of $<40 \mathrm{mg} / \mathrm{dL}$ and a triglyceride level of $>149 \mathrm{mg} / \mathrm{dL}$ or drug treatment for DL), stroke classification (TOAST classification [18]), radiological findings and the content of acute treatment/secondary prevention.

We reviewed published scientific reports by searching the PubMed database. The keywords used were "fenestration", "cerebral infarction", "basilar artery", and "vertebral artery". We reviewed related articles for all cases, and excluded "middle cerebral artery fenestration" and "double/dual vertebral artery" due to differences in symptoms, pathophysiology, and prognosis. We also excluded cases before 2000 because MRA was not common and there were mechanical differences. The same variables were collected from the previously reported cases if available (e.g., vascular risk factors, demographic data, initial treatment/final antiplatelet therapy, and MRI/MRA findings).

Fenestration classification in the basilar artery, established by Tanaka et al. [7], was used in this study, where Type I: fenestration located proximal to the AICA, Type II: bilateral AICA symmetrically originating from the fenestrated trunk, Type III: unilateral AICA originating from one side of the fenestrated trunk, and Type IV: fenestration located distal to AICA. 
Cervico-cephalic arterial dissection diagnostic criteria were as follows [19]: Major criteria: (1) ‘Double lumen' or 'intimal flap' demonstrated on either digital subtraction angiography (DSA), MRI, MRA, CTA, or duplex ultrasonography; (2) 'Pearl and string sign' or 'string sign' demonstrated on DSA; (3) Pathological confirmation of arterial dissection; Minor criteria: (4) 'Pearl sign' or 'tapered occlusion' demonstrated on DSA; (5) 'Pearl and string sign', 'string sign' or 'tapered occlusion' demonstrated on MRA; (6) 'Hyper-intense intramural signal' (corresponding to intramural hematoma) demonstrated on T1-weighted MRI; Additional criteria: (7) Change in arterial shape on either DSA, MRI, MRA, CTA or duplex ultrasonography; (8) No other causes of arterial abnormalities. Definite dissection was defined as the presence of one or more major criteria, or the presence of one or more minor criteria and both of the two additional criteria. Probable dissection was defined as the presence of one or more minor criteria.

\section{Results}

We extracted four cases from our hospital and eight from the literature search. The clinical presentation, radiological findings and vascular risk factors for the 12 cases are described in the Table 1 .

The mean age was $46.3 \pm 25.6$ years (range, $5-76$ years) and most of the patients were male (91.7\%). Half of the patients had headache (50\%). Regarding fenestration classification, type I was the most common (33.3\%). In MRA study, string shape (83.3\%) and shape change $(91.6 \%)$ were the most common. For vascular risk factors, hypertension was the most common $(50 \%)$, but no patient had diabetes $(0 \%)$. For clinical symptoms, vertigo/dizziness were the most common $(83.3 \%)$, and $33.3 \%$ patients had ataxia, limb weakness, and dysesthesia. Symptoms worsened in only one patient (8.3\%).

In our cases, radiological follow-up was performed and vascular changes were detected in all cases. As for the stroke etiology, cerebral dissection was mainly diagnosed; however, in the reported cases, vascular change was observed in seven out of eight cases, and the stroke etiology was arterial mechanical compression in one case, dissection in one case and embolic stroke of undetermined source (ESUS) in six cases. After adjusting for the dissection criteria, four of the six previously reported cases of ESUS may have been dissection.

Below, we present our four cases, which were clinically similar in terms of dissected site (in union site) and symptoms. All cases had string sign on MRA and no recurrence. 
Table 1. Clinical features, vascular risk factors and radiological findings of vertebra-basilar artery-related infarction.

\begin{tabular}{|c|c|c|c|c|c|c|c|c|c|c|c|c|c|c|c|c|c|c|c|c|c|c|}
\hline \multirow{2}{*}{ Author (et al.) } & \multirow{2}{*}{ Age } & \multirow{2}{*}{ Sex } & \multirow{2}{*}{$\begin{array}{l}\text { Stroke } \\
\text { Etiology }\end{array}$} & \multirow{2}{*}{$\begin{array}{l}\text { Fenestration } \\
\text { Classification }\end{array}$} & \multirow{2}{*}{ Headache } & \multirow{2}{*}{$\begin{array}{c}\text { Infarct } \\
\text { Area }\end{array}$} & \multicolumn{4}{|c|}{$\begin{array}{l}\text { Findings Suggesting Dissection on } \\
\text { MRA }\end{array}$} & \multirow{2}{*}{$\begin{array}{l}\text { Intramural } \\
\text { Hematoma } \\
\text { on TTWI }\end{array}$} & \multirow{2}{*}{$\begin{array}{l}\text { D-Dimer } \\
(\mu \mathrm{g} / \mathrm{mL})\end{array}$} & \multirow{2}{*}{ Hypertension } & \multirow{2}{*}{ Dyslipidemia } & \multirow{2}{*}{ Diabetes } & \multirow{2}{*}{$\begin{array}{c}\text { Atrial } \\
\text { Filiation }\end{array}$} & \multirow{2}{*}{$\begin{array}{l}\text { Related } \\
\text { Injury }\end{array}$} & \multirow{2}{*}{ Worsening } & \multirow{2}{*}{$\begin{array}{l}\text { Vertigo/ } \\
\text { Dizziness }\end{array}$} & \multirow{2}{*}{ Ataxia } & \multirow{2}{*}{ Weakness } & \multirow{2}{*}{ Dysesthesia } \\
\hline & & & & & & & String & Pearl & $\begin{array}{l}\text { Double } \\
\text { Lumen }\end{array}$ & $\begin{array}{l}\text { Shape } \\
\text { Change }\end{array}$ & & & & & & & & & & & & \\
\hline Case 1 & 71 & F & D & I & + & $\begin{array}{c}\text { pons, } \\
\text { cerebellar }\end{array}$ & + & - & - & + & - & 2.1 & + & + & - & - & - & - & + & + & - & - \\
\hline Case 2 & 74 & M & D & III & + & $\begin{array}{l}\text { Medulla } \\
\text { PICA }\end{array}$ & + & - & - & + & + & $<1.0$ & + & - & - & - & - & - & + & + & - & - \\
\hline Case 3 & 61 & M & D & III & - & cerebellar & + & - & - & + & - & 1.1 & + & - & - & - & - & - & + & - & - & - \\
\hline Case 4 & 71 & M & D & $\mathrm{I}$ & + & medulla & + & - & - & + & + & 1.5 & + & + & - & + & - & - & + & - & + & + \\
\hline Bernard [20] & 18 & M & D & (VA) & + & cerebellar & + & + & - & + & NA & NA & - & - & - & - & - & + & + & - & - & - \\
\hline Gold [16] & 12 & M & E & II & - & cerebellar & - & - & - & NA & $\mathrm{NA}$ & $\mathrm{NA}$ & - & - & - & - & - & - & + & + & - & - \\
\hline Kloska [15] & 5 & M & $\mathrm{E}$ & I & + & pons & + & - & - & + & $\mathrm{NA}$ & NA & - & - & - & - & - & - & - & - & + & - \\
\hline Meinnel [21] & 76 & $\mathrm{M}$ & E & IV & & $\begin{array}{c}\text { pons } \\
\text { corbellar }\end{array}$ & + & - & & + & NA & NA & + & + & - & - & - & - & - & - & + & + \\
\hline Palazzo [17] & 56 & M & E & I & - & $\begin{array}{l}\text { cerebellar } \\
\text { posterior } \\
\text { lobe }\end{array}$ & + & - & - & + & $\mathrm{NA}$ & NA & + & - & - & & - & - & + & + & - & - \\
\hline $\mathrm{Wu}[22]$ & 36 & M & E & IV & - & $\begin{array}{l}\text { Cerebellar } \\
\text { (vermis) }\end{array}$ & - & - & - & + & $\mathrm{NA}$ & $\mathrm{NA}$ & - & - & - & - & - & - & + & + & - & - \\
\hline Yamaguchi [23] & 45 & M & C & (VA) & & $\begin{array}{c}\text { Pons } \\
\text { cerebellar }\end{array}$ & + & - & - & + & NA & NA & - & - & - & - & - & - & + & - & - & \\
\hline Yamamoto [24] & 30 & M & E & (VA) & + & $\begin{array}{l}\text { midbrain } \\
\text { midat }\end{array}$ & + & - & - & + & NA & $<1.0$ & - & - & - & - & - & - & + & - & + & + \\
\hline
\end{tabular}

NA; not applicable, MRA; magnetic resonance angiography, D; dissection, C; compression, E; embolic stroke of undetermined source, PICA; posterior inferior cerebellar artery. 


\subsection{Case 1}

A 71-year-old female treated for dyslipidemia and hypertension developed severe headache in the left posterior region, dysarthria, vertigo, and lower limb weakness. On arrival, she had left lower limb ataxia, but no dysarthria, nystagmus or weakness on neurological examination (NIHSS 1). Brain MRI/A demonstrated a dotted acute lesion in the pons and cerebellar hemisphere (Figure 1A), reduced left vertebral artery visualization with string sign and basilar artery fenestration (Figure 1B-D). Her blood pressure was high $(193 / 80 \mathrm{mmHg}$ ) without arrythmia. Laboratory data were normal, but the FDP-D-dimer level was slightly increased $(2.1 \mathrm{mg} / \mathrm{mL})$. Cardiac/carotid echography and ECG also detected no abnormality. Based on BPAS and the presence of severe headache, we diagnosed her with cerebral infarction due to cerebral dissection, and started antiplatelet therapy and blood pressure control. After starting treatment, her symptoms and visualization of the vertebral artery improved (Figure 1E).

\subsection{Case 2}

A 66-year-old man with well-controlled hypertension felt dizziness and headache, but he stayed home. The next day, his blood pressure became high $(200 / 100 \mathrm{mmHg})$, dizziness and headache worsened, and walking became difficult. He was brought by ambulance to our hospital due to walking difficulty. His blood pressure was high $(166 / 78 \mathrm{mmHg})$ with a regular rhythm, but ECG and cardiac/carotid echography were normal. Neurological examination revealed left gaze-induced nystagmus and left limb ataxia (NIHSS 2). On MRI/A, acute infarction was detected at the left ventral medulla and left posterior cerebellar artery area (Figure 1F). Basilar artery fenestration (with intramural hematoma; Figure 1G) was found and the left vertebral artery tapered against the union of the basilar artery (Figure $1 \mathrm{H}$ ). Laboratory data were normal. We diagnosed him with cerebral infarction due to left vertebral artery dissection, and started antiplatelet therapy and blood pressure control. His symptoms and left vertebral artery visualization improved on follow-up MRA (Figure 1I).

\subsection{Case 3}

A 60-year-old male with untreated hypertension and smoking history developed headache, severe dizziness, and nausea at night. He vomited 10 times and was unable to walk. He was admitted to our emergency room. On arrival, his symptoms improved, but his blood pressure was 154/92 $\mathrm{mmHg}$ without arrhythmia. Laboratory data, ECG, and cardiac/carotid echography were normal. Neurological examination detected slight gaze-induced nystagmus (NIHSS 0). On MRI/A study, dotted acute infarction was noted in the right cerebellar area (Figure 1J), and fenestration was found on union of the right vertebral artery and basilar artery without intramural hematoma (Figure $1 \mathrm{~K}-\mathrm{M}$ ). He was diagnosed with acute cerebral infarction due to right vertebral artery dissection, and antiplatelet therapy and blood pressure control were started. On the following MRI/A, we observed the right vertebral artery, but the intimal flap was still detected in the right fenestrated area (Figure $1 \mathrm{~N}$; arrow).

\subsection{Case 4}

A 71-year-old man suddenly developed headache, nausea, and hypoesthesia in the right limbs. He had hypertension and atrial fibrillation (Af), but his adherence to medication was poor. After $3 \mathrm{~h}$ of rest, his symptoms worsened and he was admitted to our emergency room. On arrival, his blood pressure was $182 / 106 \mathrm{mmHg}$ with Af, and right central-type facial palsy and sensory disturbance (8/10) in the right limbs were detected on neurological examination (NIHSS 2). Laboratory data were normal, including FDP-D dimer levels. The left atrium was slightly dilatated on echocardiography, but carotid echography was normal. On MRI/A, a high-intensity area was detected in the left dorsal and ventral medulla (Figure 1O), and the left vertebral artery tapered off against the basilar artery with intramural hematoma, but fenestration was detected on BPAS (Figure 1P-R). We started hydration and direct oral anticoagulants (DOAC) were restarted after confirming no hemorrhagic complications on CT the next day. After starting treatment, facial palsy improved, but sensory disturbance remained. On follow-up 
MRI/A, the fenestrated vertebrobasilar artery was clearly detected, but the left fenestrated artery still had an intimal flap (Figure 1S).

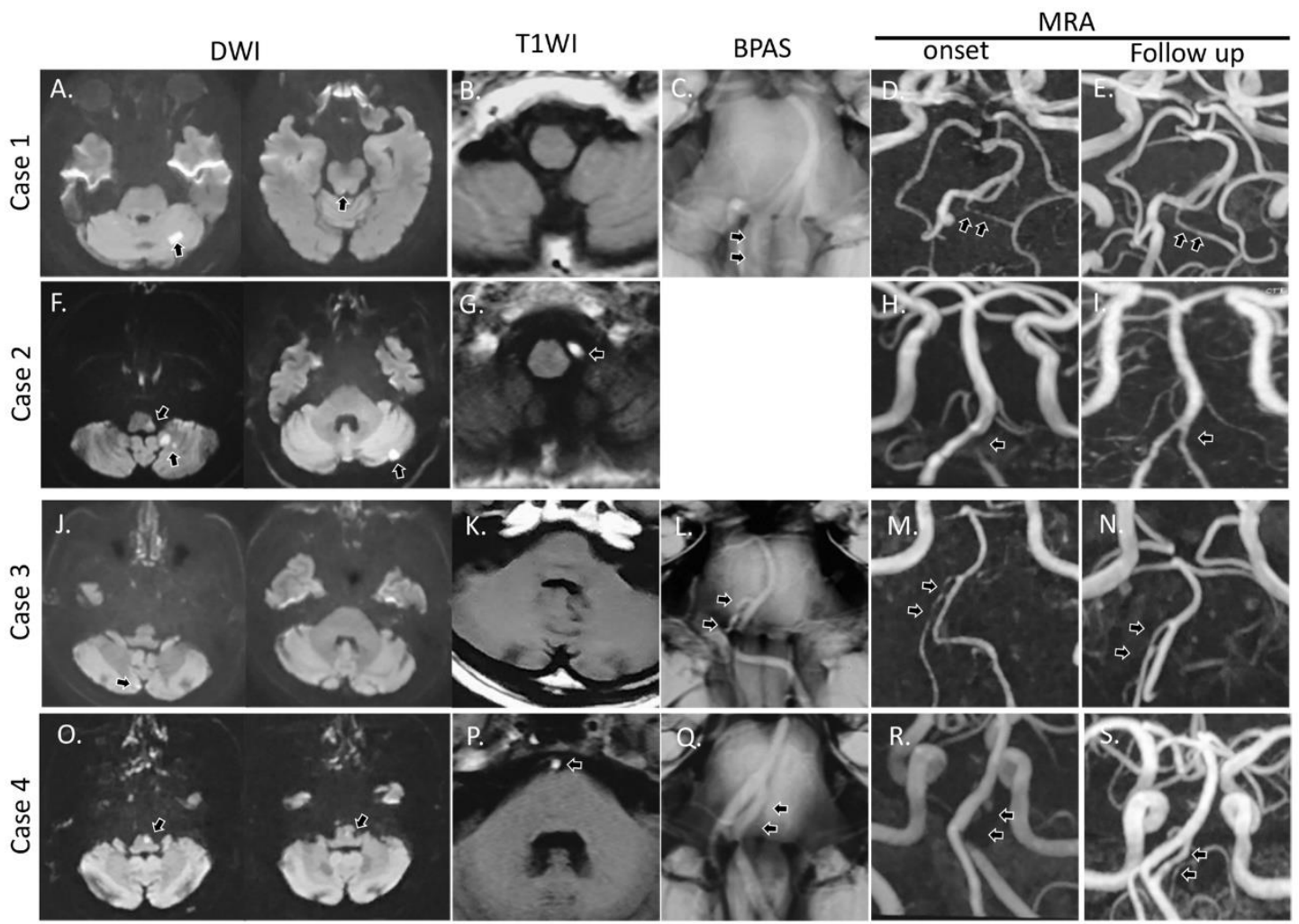

Figure 1. Magnetic resonance imaging (MRI) of our cases on diffusion weighted image (DWI; A, F, J, O), T1WI (for intramural hematoma; B, G, K, P), basi-parallel anatomic scanning magnetic resonance imaging (BPAS; C, L, Q) and magnetic resonance angiography (MRA, onset; D, H, M, R, follow-up; E, $\mathbf{I}, \mathbf{N}, \mathbf{S}$ ). Case 1; Acute infarction at the left cerebellum and dorsal pons (A; arrow), but no intramural hematoma. The left vertebral artery was observed on BPAS (C; arrow), but not on MRA (D; arrow). On follow-up MRA, the left vertebral artery was observed (E; arrow). Case 2; Acute infarction was observed in the lateral medulla and cerebellum (F; arrow). Intramural hematoma was noted at the left vertebral artery (G; arrow). On initial MRA, string sign was seen in the left vertebral artery (H; arrow). On follow-up MRA, the left vertebral artery was visualized (I; arrow). Case 3; Dotted acute infarction was noted in the right cerebellum (J; arrow), but there was no intramural hematoma (K). On BPAS and MRA, the right fenestrated artery and vertebral artery were string-shaped (L, M; arrow). On follow-up MRA, the right fenestrated artery and vertebral artery were visualized more clearly. Case 4; Acute infarction was observed at the dorsal lateral medulla (O; arrow) and intramural hematoma was present at the left fenestrated artery (P; arrow). On BPAS, the basilar artery was fenestrated ( $\mathbf{Q}$; arrow), but the left fenestrated artery disappeared on initial MRA (R; arrow). On follow-up MRA, the left fenestrated artery reappeared (S; arrow).

\section{Discussion}

The exact underlying mechanism of fenestrated-related infarction remains unknown, but it may be related to dissection and local embolism. In our cases, dissection was mainly diagnosed, but the cases found by literature search were mainly diagnosed as ESUS. This may have been due to racial differences. Common sites of dissection may differ between Asian (posterior circulation) and Caucasian (anterior circulation) populations [25]. However, among the previously reported cases, several were suspected to be dissection.

Many hypotheses have been proposed for the development of dissection/aneurysms at the junction of a fenestrated basilar artery. Elastin discontinuity with thinned sub-endothelium at the proximal end 
of the fenestration may be important for aneurysm formation, similar to cerebral artery bifurcations [26]. Based on neuropathological analysis, dissection generally disrupted the internal elastic lamina and the media, similar to aneurysms $[27,28]$. Thus, different patterns of intimal injury were found in intracranial artery dissection. Mural hematoma may be caused by one entrance in the pseudo lumen (so-called entry-only lesions) or an entrance and exit in the pseudo lumen (so-called entry-exit lesions). Entry-only lesions have a higher rate of subarachnoid hemorrhage than entry-exit dissection lesions [29]. In addition to the micro-histological factors, the hemodynamic effects, to which the arterial wall of the proximal portion in the fenestration is directly exposed, such as the shearing stress from the blood pressure, also play an important role in the development of dissection and aneurysms [25,30].

However, most fenestrations do not directly interact or associate with the dissection/aneurysm [6,27]. Fenestration and aneurysm are not particularly complex vascular anomalies, but their incidental association has been reported based on extensive examination of SAH cases [31]. In another report, 10 of 53 patients with fenestration had associated aneurysms, but none were located at the vertebrobasilar junction with basilar artery fenestration; a significant association was not established [4]. The reported incidence of coexisting fenestration and aneurysm varies from $27.5 \%$ [1] to no association [4]. However, Sun et al. [32] found that the frequency of intracranial aneurysms with fenestration was $17.0 \%$, with a significant difference compared with aneurysms unrelated to fenestration. In contrast, Gao reported that no aneurysms were located on the fenestration itself. Moreover, although no significant relationship exists between basilar artery fenestration and aneurysms, basilar artery fenestration was significantly more often associated with aneurysms of the posterior cerebral circulation [6].

According to the hydromechanical theory, pressure from blood is placed on the vessel wall during flow, involving wall shear stress and wall pressure. At the blood vessel branching site, the interior wall has high wall pressure and wall shear stress, whereas the lateral wall has low wall pressure and wall shear stress, with an irregular distribution and high pressure gradient [9]. As the atherogenic components in the blood move passively from the high-pressure area to the low-pressure area, low wall pressure is not only closely related to the blood vessel wall thickness, but also to atherosclerosis [9]. When there was a more intensive pressure change, these components significantly aggregated. Thus, hydromechanical pressure affects the vascular endothelium [33].

Our study has several limitations, including its retrospective design and the non-random treatment allocation procedure. It must be emphasized that our study was exploratory in nature and is therefore appropriate for hypothesis generation, but not for hypothesis testing. Second, we were unable to retrieve information about the duration of treatment or compliance with daily medication considering the retrospective nature of this study. Furthermore, the medical records of a substantial number of patients contained no information about long-term outcomes, or the post-discharge use of anti-platelet therapy, anti-hypertensive therapy or statins. Third, selection bias may have affected our analysis. As basilar artery occlusion affects respiration and hemodynamics, MRI was unable to be performed for those patients. Therefore, fenestration was overlooked. Indeed, in a case reported by Belly et al., fenestration was detected by post-modern analysis [34]. However, we believe that this study is useful as it may lead to treatment methods for vertebra-basilar artery fenestration-related cerebral infarction.

\section{Conclusions}

In conclusion, we reported four cases of rare fenestration-related cerebral infarction. In our cases, MRI/A analysis was performed in detail and during follow-up. Focusing on stroke etiology, cerebral artery dissection was suggested, but we were unable to exclude underlying etiologies. MRI/A and BPAS may be useful for observing the fenestrated vascular structures.

Author Contributions: Conceptualization, N.M., Y.U.; Methodology, N.M., Y.U., K.H.; Investigation, N.M., K.H., C.K., S.N.; Formal analysis, N.M.; Resources, N.M., Y.U.; Writing—original draft, N.M.; Writing-Review and editing, all authors; Supervision, K.Y., N.H. All authors have read and agreed to the published version of the manuscript.

Funding: This research received no external funding. 
Conflicts of Interest: The authors declare no conflicts of interest.

\section{References}

1. Bayrak, A.H.; Senturk, S.; Akay, H.O.; Ozmen, C.A.; Bukte, Y.; Nazaroglu, H. The frequency of intracranial arterial fenestrations: A study with 64-detector CT-angiography. Eur. J. Radiol. 2011, 77, 392-396. [CrossRef]

2. Teal, J.S.; Rumbaugh, C.L.; Bergeron, R.T.; Segall, H.D. Angiographic demonstration of fenestrations of the intradural intracranial arteries. Radiology 1973, 106, 123-126. [CrossRef]

3. Wollschlaeger, G.; Wollschlaeger, P.B.; Lucas, F.V.; Lopez, V.F. Experience and result with postmortem cerebral angiography performed as routine procedure of the autopsy. Am. J. Roentgenol. Radium. Ther. Nucl. Med. 1967, 101, 68-87. [CrossRef]

4. Bharatha, A.; Aviv, R.I.; White, J.; Fox, A.J.; Symons, S.P. Intracranial arterial fenestrations: Frequency on CT angiography and association with other vascular lesions. Surg. Radiol. Anat. 2008, 30, 397-401. [CrossRef]

5. Cademartiri, F.; Stojanov, D.; Dippel, D.W.; Van Der Lugt, A.; Tanghe, H. Noninvasive detection of a ruptured aneurysm at a basilar artery fenestration with submillimeter multisection CT angiography. AJNR Am. J. Neuroradiol. 2003, 24, 2009-2010.

6. Gao, L.Y.; Guo, X.; Zhou, J.J.; Zhang, Q.; Fu, J.; Chen, W.J.; Yang, Y.J. Basilar artery fenestration detected with CT angiography. Eur. Radiol. 2013, 23, 2861-2867. [CrossRef]

7. Tanaka, M.; Kikuchi, Y.; Ouchi, T. Neuroradiological analysis of 23 cases of basilar artery fenestration based on 2280 cases of MR angiographies. Interv. Neuroradiol. 2006, 12, 39-44. [CrossRef]

8. Zhu, X.; Liu, L.; He, X.; Zhang, X.; Hu, L.; Du, B.; Wang, W.; Jiang, W.; Liu, Z. Wall thickening pattern in atherosclerotic basilar artery stenosis. Neurol. Sci. 2016, 37, 269-276. [CrossRef]

9. Liu, L.; Zhang, X.B.; Lu, S.; Liu, Z.J.; Zhu, X.J. Plaque distribution of basilar artery fenestration by 3D High-resolution MR vessel wall imaging. Cell Transplant. 2019, 28, 851-855. [CrossRef]

10. Campos, J.; Fox, A.J.; Vinuela, F.; Lylyk, P.; Ferguson, G.G.; Drake, C.G.; Peerless, S.J. Saccular aneurysms in basilar artery fenestration. AJNR Am. J. Neuroradiol. 1987, 8, 233-236.

11. Peluso, J.P.; van Rooij, W.J.; Sluzewski, M.; Beute, G.N. Aneurysms of the vertebrobasilar junction: Incidence, clinical presentation, and outcome of endovascular treatment. AJNR Am. J. Neuroradiol. 2007, 28, 1747-1751. [CrossRef]

12. Rinkel, G.J. Natural history, epidemiology and screening of unruptured intracranial aneurysms. J. Neuroradiol. 2008, 35, 99-103. [CrossRef]

13. Bozek, P.; Pilch-Kowalczyk, J.; Kluczewska, E.; Zymon-Zagorska, A. Detection of cerebral artery fenestrations by computed tomography angiography. Neurol. Neurochir. Pol. 2012, 46, 239-244. [CrossRef]

14. Kachhara, R.; Nair, S.; Gupta, A.K. Fenestration of the proximal anterior cerebral artery (A1) with aneurysm manifesting as subarachnoid hemorrhage-Case report. Neurol. Med. Chir. (Tokyo) 1998, 38, 409-412. [CrossRef]

15. Kloska, S.P.; Schlegel, P.M.; Strater, R.; Niederstadt, T.U. Causality of pediatric brainstem infarction and basilar artery fenestration? Pediatr. Neurol. 2006, 35, 436-438. [CrossRef]

16. Gold, J.J.; Crawford, J.R. An unusual cause of pediatric stroke secondary to congenital basilar artery fenestration. Case Rep. Crit. Care 2013, 2013, 627972. [CrossRef]

17. Palazzo, P.; Ruff, M.; Lyerly, M.J.; Alexandrov, A.V. Basilar artery thrombus vs. fenestration: A differential diagnostic challenge in acute ischemic stroke. J. Neuroimaging 2014, 24, 607-609. [CrossRef]

18. Adams, H.P., Jr.; Bendixen, B.H.; Kappelle, L.J.; Biller, J.; Love, B.B.; Gordon, D.L.; Marsh, E.E., 3rd. Classification of subtype of acute ischemic stroke. Definitions for use in a multicenter clinical trial. TOAST. Trial of Org 10172 in Acute Stroke Treatment. Stroke 1993, 24, 35-41.

19. Sato, S.; Toyoda, K.; Matsuoka, H.; Okatsu, H.; Kasuya, J.; Takada, T.; Shimode, A.; Uehara, T.; Naritomi, H.; Minematsu, K. Isolated anterior cerebral artery territory infarction: Dissection as an etiological mechanism. Cerebrovasc. Dis. 2010, 29, 170-177. [CrossRef]

20. Bernard, T.J.; Mull, B.R.; Handler, M.H.; Harned, R.K.; Filley, C.M.; Kumpe, D.A.; Tseng, B.S. An 18-year-old man with fenestrated vertebral arteries, recurrent stroke and successful angiographic coiling. J. Neurol. Sci. 2007, 260, 279-282. [CrossRef]

21. Meinel, T.R.; Pult, F.; Gralla, J.; Arnold, M.; Bassetti, C.; Jung, S. Successful endovascular recanalization of a partially occluded basilar artery fenestration. Interv. Neuroradiol. 2019, 25, 44-46. [CrossRef] 
22. Wu, X.; Lin, A.; Zhu, J.; Cai, B. Basilar artery fenestration: An unusual possible cause of ischaemic stroke? BMJ Case Rep. 2018, 2018. [CrossRef]

23. Yamaguchi, S.; Horie, N.; Tsunoda, K.; Tateishi, Y.; Izumo, T.; Hayashi, K.; Tsujino, A.; Nagata, I. Bow hunter's stroke due to stretching of the vertebral artery fenestration: A case report. NMC Case Rep. J. 2015, 2, 9-11. [CrossRef]

24. Yamamoto, R.; Koyama, K.; Kurokawa, T.; Kurosawa, Y. Brainstem infarction due to vertebral artery fenestration in a young adult man: A case report. Jpn. J. Stroke 2010, 32, 174-178. [CrossRef]

25. Debette, S.; Compter, A.; Labeyrie, M.A.; Uyttenboogaart, M.; Metso, T.M.; Majersik, J.J.; Goeggel-Simonetti, B.; Engelter, S.T.; Pezzini, A.; Bijlenga, P.; et al. Epidemiology, pathophysiology, diagnosis, and management of intracranial artery dissection. Lancet Neurol. 2015, 14, 640-654. [CrossRef]

26. Finlay, H.M.; Canham, P.B. The layered fabric of cerebral artery fenestrations. Stroke 1994, 25, $1799-1806$. [CrossRef]

27. Ono, H.; Nakatomi, H.; Tsutsumi, K.; Inoue, T.; Teraoka, A.; Yoshimoto, Y.; Ide, T.; Kitanaka, C.; Ueki, K.; Imai, H.; et al. Symptomatic recurrence of intracranial arterial dissections: Follow-up study of 143 consecutive cases and pathological investigation. Stroke 2013, 44, 126-131. [CrossRef]

28. Ro, A.; Kageyama, N.; Abe, N.; Takatsu, A.; Fukunaga, T. Intracranial vertebral artery dissection resulting in fatal subarachnoid hemorrhage: Clinical and histopathological investigations from a medicolegal perspective. J. Neurosurg. 2009, 110, 948-954. [CrossRef]

29. Mizutani, T.; Kojima, H.; Asamoto, S.; Miki, Y. Pathological mechanism and three-dimensional structure of cerebral dissecting aneurysms. J. Neurosurg. 2001, 94, 712-717. [CrossRef]

30. Tsuei, Y.S.; Matsumoto, Y.; Ohta, M.; Nakayama, T.; Ezura, M.; Takahashi, A. Vertebrobasilar junction fenestration with dumbbell-shaped aneurysms formation: Computational fluid dynamics analysis. Surg. Neurol. 2009, 72 (Suppl. 2), S11-S19. [CrossRef]

31. Tanaka, M.; Matsumoto, S. [Fenestration of the internal carotid artery (author's transl)]. Neurol. Med. Chir. (Tokyo) 1982, 22, 291-294. [CrossRef]

32. Sun, Z.K.; Li, M.; Li, M.H.; Li, Y.D.; Sun, W.P.; Zhu, Y.Q. Fenestrations accompanied by intracranial aneurysms assessed with magnetic resonance angiography. Neurol. India 2012, 60, 45-49. [CrossRef]

33. Giannoglou, G.D.; Soulis, J.V.; Farmakis, T.M.; Farmakis, D.M.; Louridas, G.E. Haemodynamic factors and the important role of local low static pressure in coronary wall thickening. Int. J. Cardiol. 2002, 86, 27-40. [CrossRef]

34. Berry, A.D., 3rd; Kepes, J.J.; Wetzel, M.D. Segmental duplication of the basilar artery with thrombosis. Stroke 1988, 19, 256-260. [CrossRef]

(C) 2020 by the authors. Licensee MDPI, Basel, Switzerland. This article is an open access article distributed under the terms and conditions of the Creative Commons Attribution (CC BY) license (http://creativecommons.org/licenses/by/4.0/). 